\title{
Nonlinear optical beams in bounded nematic liquid crystal cells
}

\author{
Antonmaria A. Minzoni ${ }^{1} \quad$ Luke W. Sciberras $^{2}$ \\ Noel F. Smyth ${ }^{3} \quad$ Annette L. Worthy ${ }^{4}$
}

(Received 5 January 2012; revised 14 June 2012)

\begin{abstract}
Stable nonlinear beams, both solitary waves (nematicons) and optical vortices, can form in a nematic liquid crystal due to a balance between the nonlinear, nonlocal response of the nematic and the diffractive spreading of the light beam. The 'huge' nonlinearity of a nematic liquid crystal makes it ideal for the experimental development of photonic devices as nonlinear effects occur over millimetre distances. In this work, a simple and fast method to analyse the trajectory of a nonlinear beam within a finite liquid crystal cell, based on a classical method not explored in this context, the method of images, is developed. With the orientation of the nematic molecules modelled using images, the evolution of the beam is obtained by using both asymptotics and modulation theory. The efficiency of this new method is shown by comparisons with a standard Fourier series solution for the nematic
\end{abstract}

http://journal.austms.org.au/ojs/index.php/ANZIAMJ/article/view/5076 gives this article, (c) Austral. Mathematical Soc. 2012. Published July 15, 2012. ISSN 1446-8735. (Print two pages per sheet of paper.) Copies of this article must not be made otherwise available on the internet; instead link directly to this URL for this article. 
response and full numerical solutions of the governing equations. It is found that only a small number of images is required compared with the usual Fourier series technique in order to obtain excellent agreement with full numerical solutions. Finally, the contrasting effect of the cell boundaries on a nematicon and a vortex is explored.

\section{Contents}

1 Introduction

2 Modulation theory

3 Optical vortex

4 Results and discussion

References
C374

C375

C381

C381

C384

\section{Introduction}

The propagation of light in liquid crystals has captured the attention of experimentalists for decades. The nematic phase of a liquid crystal is ideal for the propagation of nonlinear beams due to its 'huge' nonlinearity, so that nonlinear effects are observed over millimetre distances. Nematic liquid crystals (NLC) also have a 'nonlocal' response, so that the effect of an optical beam on the nematic extends far beyond the beam waist. This nonlocal response stops the collapse of 2D bulk solitary waves [5, 6, 11], termed 'nematicons', and optical vortices $[14,16]$. Experiments conducted using NLC have shown the medium to be advantageous for use in all-optical devices, including circuits and logic gates $[4,5,10]$, due to the possibility for reconfigurable waveguides. To overcome the Freédericksz threshold [9] the nematic molecules are pre- 
tilted, which is done by either applying an external static electric field to the cell $[5,6]$ or by 'rubbing' the cell walls to induce an alignment of the nematic molecules at the walls, which is then propagated into the bulk of the cell due to elastic forces $[1,3]$. The second mechanism is considered here.

This work contrasts the evolution of a nematicon and an optical vortex as they propagate down a cell. This evolution is studied using a hybrid technique based on an exact solution for the nematic response and a Lagrangian formulation, termed modulation theory [15], for the optical beam. The nematic response is found from a Green's function solution derived using the method of images (MoI) [7]. The Green's function solution is shown to be equivalent to the usual Fourier series solution, but is superior as far less terms are needed to obtain good agreement with full numerical solutions. A further advantage of the approximate technique of the present work is that it includes the effect of the diffractive radiation shed by the nematicon as it evolves $[8,13]$. It is found that the cell boundaries do not affect the stability of a nematicon, but can excite the mode 2 azimuthal instability $[14,16]$ of an optical vortex.

\section{Modulation theory}

A linearly polarised coherent light beam is inputted into a planar nematic liquid crystal cell. We take the $z$ direction as the beam propagation direction, the $x$ direction as the direction of beam polarisation, with $y$ completing the coordinate system. A nonlinear beam then occurs due to a balance between linear diffraction and the self-focusing of the beam. To obtain a beam at milliwatt power levels, the nematic molecules are pre-tilted at an angle $\theta_{0} \sim \pi / 4$ to the $z$ direction by rubbing of the cell walls, thus eliminating the Freédericksz threshold [6, 9]. The non-dimensional equations governing the propagation of the nematicon in the paraxial approximation are $[1,2,3]$

$$
i \frac{\partial \mathrm{E}}{\partial z}+\frac{1}{2} \nabla^{2} \mathrm{E}+2 \mathrm{E} \theta=0, \quad v \nabla^{2} \theta=-2|\mathrm{E}|^{2} .
$$


Here the Laplacian $\nabla^{2}$ is in the $(x, y)$ plane. The left-hand equation of (1) is a nonlinear Schrödinger-type equation governing the electric field $E$ of the light beam, with $z$ being a time-like coordinate. The right-hand equation of (1) is the director (a Poisson) equation which governs the perturbation $\theta$ of the nematic molecules from the pre-tilt angle $\theta_{0}$. The parameter $v$ is the intermolecular elasticity parameter of the liquid crystal, with large values corresponding to a nonlocal response. In experiments $v=\mathrm{O}(100)$ [6]. The geometry of the cell is rectangular, $0 \leqslant x \leqslant L_{x}$ and $0 \leqslant y \leqslant L_{y}$. The anchoring conditions give $\theta=0$ at $x=0, L_{x}$ and $y=0, L_{y}$. The director equation in (1) can be solved using a Green's function, $G\left(x, y ; x^{\prime}, y^{\prime}\right)$, with

$$
\theta=-\frac{2}{v} \int_{0}^{L_{y}} \int_{0}^{L_{x}} G\left(x, y ; x^{\prime}, y^{\prime}\right)\left|E\left(x^{\prime}, y^{\prime}\right)\right|^{2} d x^{\prime} d y^{\prime} .
$$

The electric field equation in (1) then has the Lagrangian

$$
L=i\left(E^{*} E_{z}-E E_{z}^{*}\right)-|\nabla E|^{2}-\frac{4}{v}|E|^{2} \int_{0}^{L_{y}} \int_{0}^{L_{x}} G\left(x, y ; x^{\prime}, y^{\prime}\right)\left|E\left(x^{\prime}, y^{\prime}\right)\right|^{2} d x^{\prime} d y^{\prime},
$$

where the superscript ${ }^{*}$ denotes the complex conjugate.

Let us first consider the evolution of a nematicon. The nematicon equations (1) have no known exact solitary wave solution. A hybrid method based on Lagrangian techniques [15] and exact solutions is developed and studied here. This is based on the Gaussian trial function

$$
E=\left(a e^{-r^{2} / w^{2}}+i g\right) e^{i \phi}
$$

for the nematicon profile $[8,12]$. Here $r^{2}=(x-\xi)^{2}+(y-\eta)^{2}$ and $\phi=$ $\sigma+V_{x}(x-\xi)+V_{y}(y-\eta)$. The amplitude $a$, width $w$, phase $\sigma$, position $(\xi, \eta)$, velocity $\left(V_{x}, V_{y}\right)$ and $g$ are all functions of $z$. The trial function is circularly symmetric. In experiments the beam has been measured to have a slight elliptic cross section [4]. The first term in the trial function is a varying solitary wave-like beam. The second term is related to the shelf of low wavenumber diffractive radiation that accumulates under the evolving nematicon $[8,13]$. 
This shelf is $\pi / 2$ out of phase with the nematicon [8]. The Green's function for the director, right-hand equation in (1) is [7]

$$
G\left(x, y ; x^{\prime}, y^{\prime}\right)=-\frac{1}{2 \pi} \ln f\left(x, y, x^{\prime}, y^{\prime}\right) .
$$

The Green's function is constructed by reflections of the point source at $\left(x^{\prime}, y^{\prime}\right)$ in the cell walls, symmetrically creating a series of positive (source) and negative (sink) images across the lattice in order to satisfy the zero boundary conditions. This process is illustrated in Figure 1.

As the function $f$ in (5) has only simple poles and simple zeros, the simplest analytical expression for $f$ is in terms of elliptic functions [7]

$$
f(x, y)=\frac{\sigma(z-\zeta) \sigma(z+\zeta)}{\sigma\left(z-\zeta^{*}\right) \sigma\left(z+\zeta^{*}\right)}, \quad \sigma(z)=z \prod_{\omega \neq 0}\left[\left(1-\frac{z}{2 \omega}\right) e^{z / 2 \omega+z^{2} / 8 \omega^{2}}\right]
$$

with $z=x+i y, \zeta=x^{\prime}+i y^{\prime}$ and $\omega=n L_{x}+i m L_{y}$. In the nonlocal limit with $v$ large the beam is much narrower than the director response. Hence, the beam $|\mathrm{E}|^{2}$ in (2) is approximated by a delta function, resulting in

$$
\theta=\frac{a^{2} w^{2}}{4 v} \mathfrak{R}\left\{\frac{\sigma(z-\psi) \sigma(z+\psi)}{\sigma\left(z-\psi^{*}\right) \sigma\left(z+\psi^{*}\right)}\right\}, \quad \text { where } \quad \psi=\xi+i \eta
$$

The Green's function can also be expressed in terms of a Fourier sine series. The Green's function $G\left(x, y ; x^{\prime}, y^{\prime}\right)$ satisfies $\nabla^{2} G\left(x, y ; x^{\prime}, y^{\prime}\right)=\delta\left(x-x^{\prime}\right) \delta(y-$ $\left.y^{\prime}\right)$ with homogeneous boundary conditions. It can then be found to be

$$
\mathrm{G}\left(x, y ; x^{\prime}, y^{\prime}\right)=\frac{4}{\pi^{2} \mathrm{~L}_{x} \mathrm{~L}_{y}} \sum_{n, m=1}^{\infty} \phi_{\mathfrak{n}}(\xi) \phi_{\mathfrak{m}}(\eta) \phi_{\mathfrak{n}}(x) \phi_{\mathfrak{m}}(y)\left(\frac{\mathrm{n}^{2}}{\mathrm{~L}_{x}^{2}}+\frac{\mathrm{m}^{2}}{\mathrm{~L}_{y}^{2}}\right)^{-1},
$$

where the eigenfunctions are $\phi_{n}(x)=\sin \left(n \pi x / L_{x}\right)$ and $\phi_{m}(y)=\sin \left(m \pi y / L_{y}\right)$. Substitution of the trial function (4) for the optical beam and the director solution (7) into the Lagrangian (3) and averaging by integrating in $x$ and $y$ 


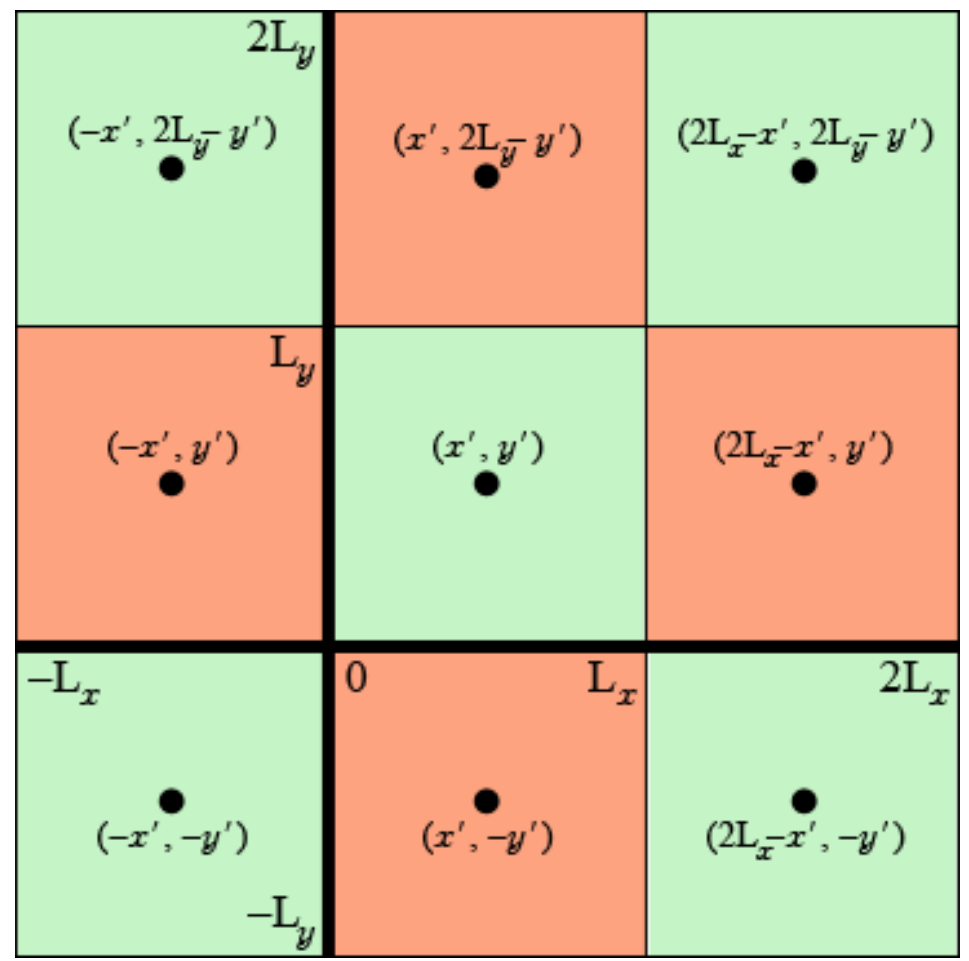

Figure 1: Method of images, depicting the sources (red or dark squares) and sinks (green or light squares) over the constructed lattice for the first eight images.

over the cell gives the averaged Lagrangian [15]

$$
\begin{aligned}
\mathcal{L}= & -2\left(\frac{1}{4} a^{2} w^{2}+\Lambda g^{2}\right)\left(\sigma^{\prime}-V_{x} \xi^{\prime}-V_{y} \eta^{\prime}+\frac{1}{2} V_{x}^{2}+\frac{1}{2} V_{y}^{2}\right)-a w^{2} g^{\prime} \\
& +g w^{2} a^{\prime}+2 a g w w^{\prime}-\frac{a^{2}}{2}+\frac{a^{4} w^{4}}{4 v}\left[-\Delta_{1}-\Delta_{2}+\Delta_{3}+\Delta_{4}\right] .
\end{aligned}
$$


Here

$$
\begin{aligned}
\Delta_{1}= & \ln \frac{w}{\sqrt{2}}-\frac{\gamma}{2}-\ln 2+\ln \sqrt{\xi^{2}+\eta^{2}}-\ln (\xi \eta), \\
\Delta_{2}= & \sum_{n, m=-\infty}^{\infty}\left[\frac{1}{2} \ln \left(\frac{\left(n L_{x}-\xi\right)^{2}+\left(m L_{y}-\eta\right)^{2}}{n^{2} L_{x}^{2}+m^{2} L_{y}^{2}}\right)\right. \\
& \left.+\frac{\left(\xi^{2}-\eta^{2}\right)\left(n^{2} L_{x}^{2}-m^{2} L_{y}^{2}\right)+4 n m \xi \eta L_{x} L_{y}}{2\left(n^{2} L_{x}^{2}+m^{2} L_{y}^{2}\right)^{2}}\right], \\
\Delta_{3}= & \sum_{n, m=-\infty}^{\infty}\left[\frac{1}{2} \ln \left(\frac{n^{2} L_{x}^{2}+\left(m L_{y}-\eta\right)^{2}}{n^{2} L_{x}^{2}+m^{2} L_{y}^{2}}\right)+\frac{\eta^{2}\left(n^{2} L_{x}^{2}-m^{2} L_{y}^{2}\right)}{2\left(n^{2} L_{x}^{2}+m^{2} L_{y}^{2}\right)^{2}}\right], \\
\Delta_{4}= & \sum_{n, m=-\infty}^{\infty}\left[\frac{1}{2} \ln \left(\frac{\left(n L_{x}-\xi\right)^{2}+m^{2} L_{y}^{2}}{n^{2} L_{x}^{2}+m^{2} L_{y}^{2}}\right)+\frac{\xi^{2}\left(n^{2} L_{x}^{2}-m^{2} L_{y}^{2}\right)}{2\left(n^{2} L_{x}^{2}+m^{2} L_{y}^{2}\right)^{2}}\right],
\end{aligned}
$$

where $\gamma$ is Euler's constant, $\gamma=0.577215665$. Taking variations of this averaged Lagrangian with respect to the nematicon parameters gives the modulation equations for the evolution of the beam. One major effect of non-locality is to shift the point at which the nematicon sheds diffractive radiation from the edge of the shelf to a new radius $\tilde{\ell}$ from the nematicon position $(\xi, \eta)$, this being the edge of the director response, termed the outer shelf radius [13]. In the present case of a finite cell the director response extends to the cell walls, so that the diffractive radiation is shed in a boundary layer at the cell walls. Hence $\tilde{\ell}=\min \left(\mathrm{L}_{x} / 2, \mathrm{~L}_{y} / 2\right)$ and $\tilde{\Lambda}=\tilde{\ell}^{2} / 2$.

The modulation equation for $\mathrm{g}$ (not shown) can be used to find the relationship between the steady state amplitude $\hat{a}$ and width $\hat{w}, \hat{a}^{2}=4 v / \widehat{w}^{4}$. Nöther's Theorem can then be used on the Lagrangian (3) to find the energy conservation equation for the nematicon, from which the steady state can be determined from the input beam. The length of the shelf of radiation under the beam is $\ell=3 \beta \pi^{2} \hat{w} / 8[8,12]$, where $\beta$ is constant. The choice $\beta=0.4$ gives good agreement with the period of numerical solutions [12]. 

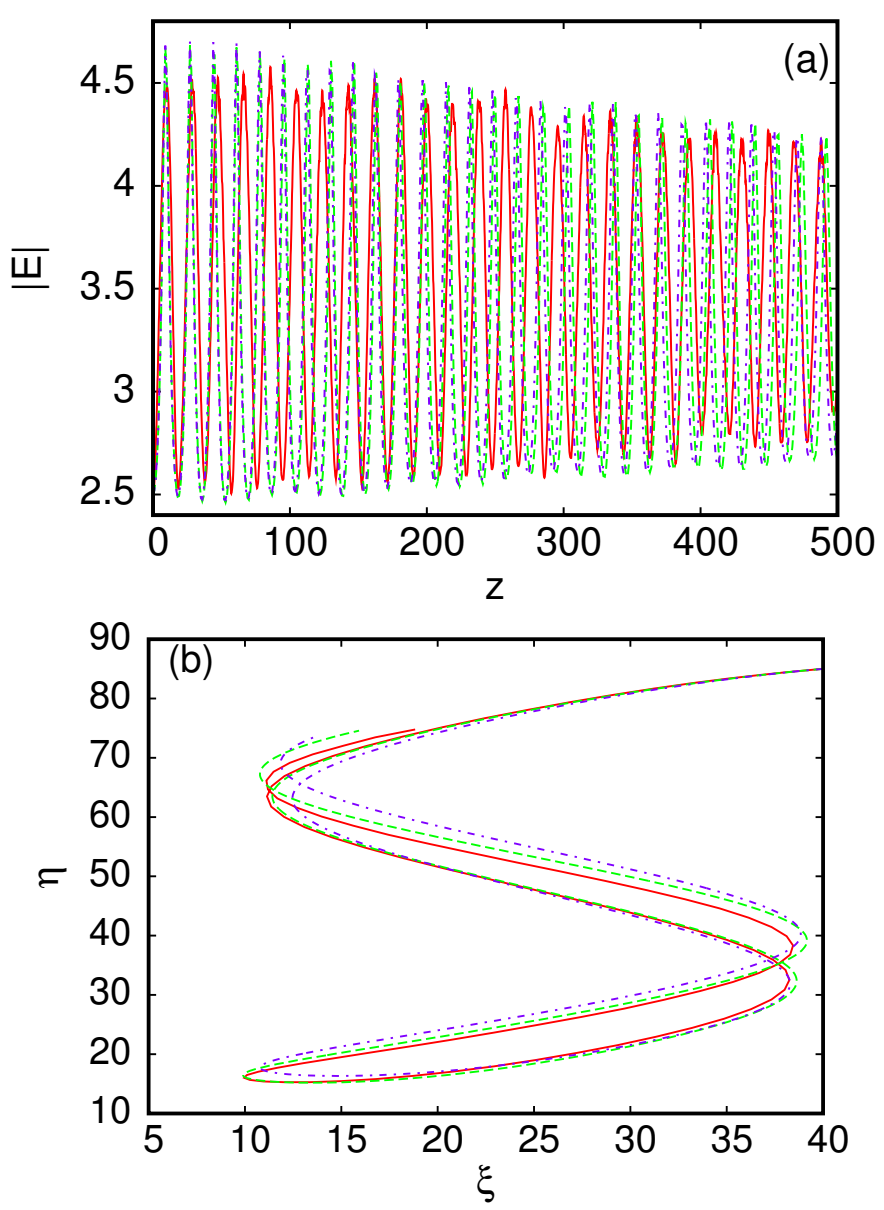

Figure 2: Comparison between: the full numerical solution, —— (full, red line); method of images solution, $-\ldots+\ldots$ (dashed, green line); and images solution with eight images, $\cdot-\cdot \cdot \cdot \cdot \cdot-\cdot-$ (dot-dashed, blue line); for the (a) amplitude $a$ and (b) $x-y$ position for a rectangular cell. The initial values are $a=2.5, w=4, \xi=40, \eta=85, V_{x}=0$ and $V_{y}=0$ with $v=200, \mathrm{~L}_{x}=50$ and $\mathrm{L}_{y}=100$. 


\section{Optical vortex}

Let us now consider the evolution of an optical vortex, which is a nonlinear beam with a $2 n \pi$ phase increase, $n$ an integer, around a central phase singularity at which the beam amplitude is zero. The cell configuration is the same as that for the nematicon of Section 2. The governing equations for the electric field envelope and the director response (1) remain the same as those for the nematicon. An optical vortex has a symmetry breaking mode 2 azimuthal instability [11]. However, in an unbounded cell, sufficiently large values of the non-locality $v, v=\mathrm{O}(100)$, stabilise the vortex $[14,16]$. A modulation theory for the evolution of an optical vortex is developed in an analogous fashion to that for a nematicon in Section 2, based on the vortex trial function

$$
E=a r e^{-r^{2} / w^{2}} e^{i(\phi+\varphi)}+i g e^{i(\phi+\varphi)},
$$

where the definitions correspond to those presented in Section 2, with the exception that $\varphi$ is the polar angle relative to the vortex centre $r=0$. The major difference for a vortex in the present finite cell to previous work is that interaction with the cell walls can destabilise the vortex, even for values of $v$ for which the vortex is stable away from the walls.

\section{Results and discussion}

The modulation equations for the nematicon were solved numerically using the standard fourth order Runge-Kutta method. These solutions were then compared with full numerical solutions of the nematicon equations (1). The electric field equation in (1) was solved using standard, second order, centred differences, for the Laplacian $\nabla^{2} \mathrm{E}$ and a second order, predictor-corrector method based upon the second order Runge-Kutta method to advance in $z$, with the initial condition given by the trial function (4) for the electric field $E$ with $g=0$. The Laplacian $\nabla^{2} \theta$ for the director equation in (1) was again approximated using second order, centred differences, and Jacobi iteration was 

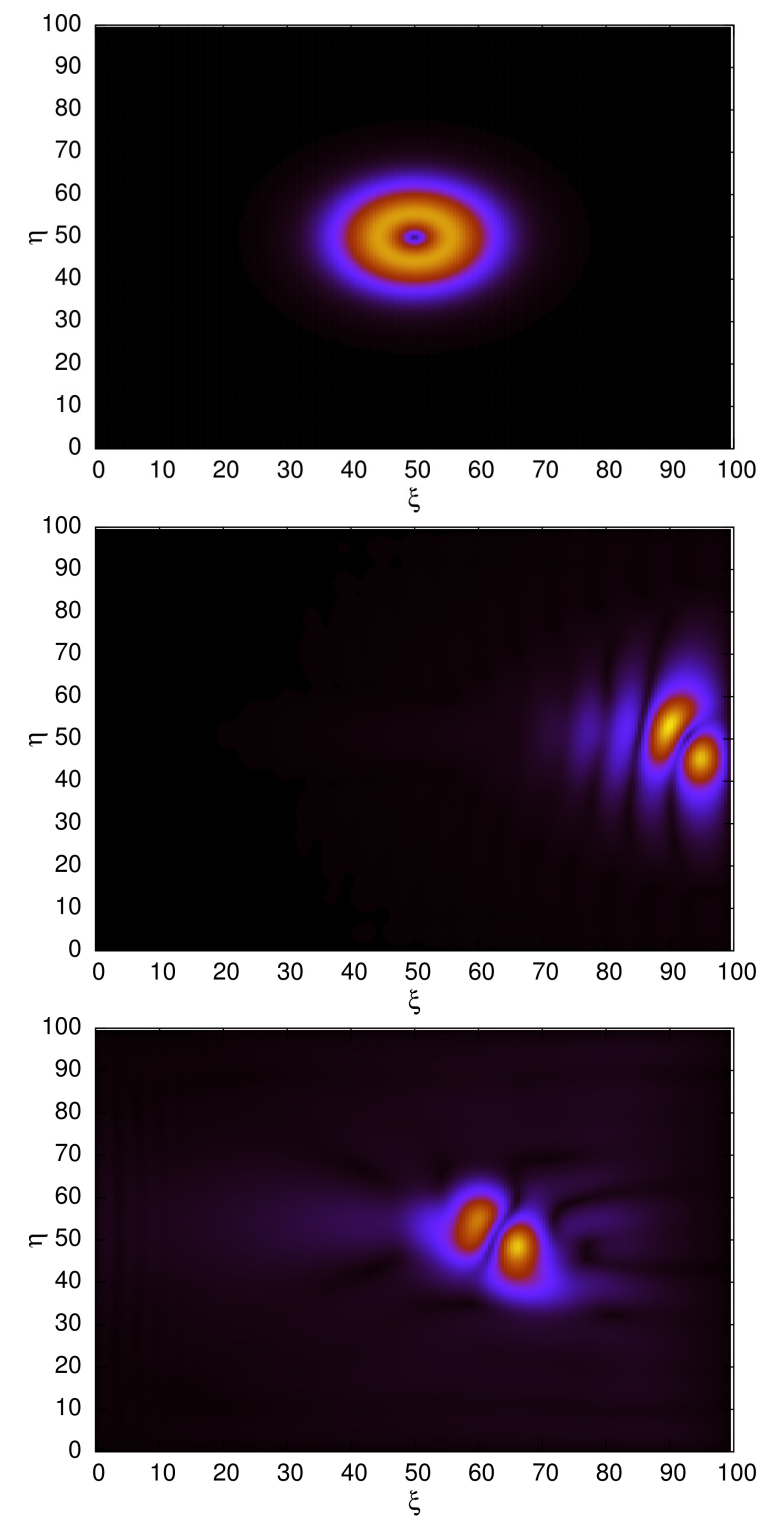

Figure 3: Full numerical solutions for a vortex. From top to bottom $z=0$, $z=100$ and $z=170$. The initial values are $a=0.15, w=8, \xi=50$, $\eta=50, V_{x}=0.6$ and $V_{y}=0$ in (14) with $v=200, L_{x}=100$ and $L_{y}=100$. 
used to solve the resulting linear system. The step sizes $\Delta x=\Delta y=0.2$ and $\Delta z=0.001$ were used. The simulation of a nematic cell with a rectangular cross-section mimicking the experimental set-up [1,3] was considered with a typical non-dimensional non-locality parameter value $v=200$. The full numerical scheme for the optical vortex was the same as that for the nematicon, but the initial condition for the electric field was given by (14) with $\mathrm{g}=0$.

Figure 2 shows the excellent agreement of the MoI solution with only eight image points with the full numerical solution, indicating the efficiency of using the method of images to calculate the nematic response. The nematicon's position evolution is shown in Figure 2(b), and again excellent agreement with the numerical solution is seen. These results show that the method of images can provide an important tool for experimental studies, as the hybrid solution is easily applicable to many other scenarios and requires minimal CPU time in comparison with full numerical solutions. The nematicon 'bounces' around the cell as it propagates, as shown in Figure 2(b). This spiralling behaviour is linked to the repulsive nature of the cell walls acting on the nematicon $[1,3]$. Away from the cell walls, for $v=200$, as used here, an optical vortex is stable. For low values of the initial speed $\sqrt{V_{x}^{2}+V_{y}^{2}}$ the vortex remains stable and has the same spiralling trajectory down the cell as for a nematicon, with similar excellent agreement between the modulation and numerical solutions. Increasing the initial speed forces the vortex closer to the cell walls. At sufficiently close approach for sufficiently high initial speed, the interaction with the boundary excites the mode 2 azimuthal instability $[14,16]$ and the vortex breaks up into two nematicons. For instance, if the vortex is initially propagated in the $x$ direction, for velocities $V_{x}<V_{\text {thr }}$ the vortex bounces off the cell wall. However, if $V_{x} \geqslant V_{\text {thr }} \sim 0.6$ the close interaction with the cell walls excites the mode 2 instability, causing the vortex to pinch off symmetrically and so split into two nematicons which rotate around a shared centre of mass due to conservation of angular momentum. This process is illustrated in Figure 3 for $V_{x}=0.6$. Numerical solutions indicate that the critical initial speed is related to the width of the initial vortex. 
In this article the effect of cell boundaries on a nematicon and an optical vortex propagating in a finite nematic liquid crystal cell was studied. A hybrid technique was used to find an approximate solution of the nematicon equations (1) with the director equation in (1) being solved exactly using the MoI to construct a Green's function solution. The MoI Green's function was shown to be equivalent to the usual Fourier series eigenfunction expansion. The advantages of the present technique were illustrated by comparisons with full numerical solutions. Not only is the technique straightforward in its application, it is also computationally very efficient. This is due to very few images being needed to provide an accurate solution. The present technique can be extended to many other cell configurations for which the director equation can be solved using the MoI. The fundamental differences between nematicon and vortex evolution in a finite cell were also illustrated.

Acknowledgements We thank CSIRO/ANZIAM student support scheme for supporting Luke Sciberras to attend the EMAC conference.

\section{References}

[1] A. Alberucci and A. Assanto, Propagation of optical spatial solitons in finite-size media: interplay between nonlocality and boundary conditions, J. Opt. Soc. Amer. B, 24, 2314-2320 (2007), doi:10.1364/JOSAB.24.002314. C375, C383

[2] A. Alberucci, G. Assanto, D. Buccoliero, A. S. Desyatnikov, T. R. Marchant, and N. F. Smyth, Modulation analysis of boundary-induced motion of optical solitary waves in a nematic liquid crystal, Phys. Rev. A, 79, 043816 (2009), doi:10.1103/PhysRevA.79.043816. C375

[3] A. Alberucci, M. Peccianti and G. Assanto, Nonlinear bouncing of nonlocal spatial solitons at boundaries, Opt. Lett., 32, 2795-2797 (2007), doi:10.1364/OL.32.002795. C375, C383 
[4] G. Assanto, A. Fratalocchi and M. Peccianti, Spatial solitons in nematic liquid crystals: from bulk to discrete, Opt. Express,, 15, 5248-5259 (2007), doi:10.1364/OE.15.005248. C374, C376

[5] G. Assanto, M. Peccianti and C. Conti, Nematicons: optical spatial solitons in nematic liquid crystal, Opt. Photon. News, 14, 44-48 (2003), doi:10.1364/OPN.14.2.000044. C374, C375

[6] C. Conti, M. Peccianti, G. Assanto, Route to nonlocality and observation of accessible solitons, Phys. Rev. Lett., 91, 073901 (2003), doi:10.1103/PhysRevLett.91.073901. C374, C375, C376

[7] R. Courant and D. Hilbert, Methods of Mathematical Physics Vol. 1, Interscience Publishers, New York (1965). C375, C377

[8] W. L. Kath and N. F. Smyth, Soliton evolution and radiation loss for the nonlinear Schrödinger equation, Phys. Rev. E, 51, 1484-1492 (1995), doi:10.1103/PhysRevE.51.1484. C375, C376, C377, C379

[9] I. C. Khoo, Liquid Crystals: Physical Properties and Nonlinear Optical Phenomena, Wiley, New York (1995). C374, C375

[10] I. C. Khoo, Nonlinear optics of liquid crystalline materials, Phys. Rep., 471, 221-267 (2009), doi:10.1016/j.physrep.2009.01.001. C374

[11] Yu. S. Kivshar and G. Agrawal, Optical Solitons: From Fibers to Photonic Crystals, Academic Press, San Diego, (2003). C374, C381

[12] A. A. Minzoni, L. W. Sciberras, N. F. Smyth and A. L. Worthy, Propagation of optical spatial solitary waves in bias-free nematic-liquid-crystal cells, Phys. Rev. A, 84, 043823 (2011), doi:10.1103/PhysRevA.84.043823. C376, C379

[13] A. A. Minzoni, N. F. Smyth and A. L. Worthy, Modulation solutions for nematicon propagation in nonlocal liquid crystals, J. Opt. Soc. Amer. B, 24, 1549-1556 (2007), doi:10.1364/JOSAB.24.001549. C375, C376, C379 
[14] A. A. Minzoni, N. F. Smyth, A. L. Worthy and Yu. S. Kivshar, Stabilization of vortex solitons in nonlocal nonlinear media, Phys. Rev. A, 76, 063803 (2007), doi:10.1103/PhysRevA.76.063803. C374, C375, C381, C383

[15] G. B. Whitham, Linear and Nonlinear Waves, J. Wiley and Sons, New York, (1974). C375, C376, C378

[16] A. I. Yakimenko, Yu. A. Zaliznyak and Yu. S. Kivshar, Stable vortex solitons in nonlocal self-focusing nonlinear media, Phys. Rev. E, 71, 065603(R) (2005), doi:10.1103/PhysRevE.71.065603. C374, C375, C381, C383

\section{Author addresses}

1. Antonmaria A. Minzoni, Fenomenos Nonlineales y Mecánica (FENOMEC), Department of Mathematics and Mechanics, Instituto de Investigación en Matemáticas Aplicádas y Sistemas, Universidad Nacional Autónoma de México, 01000 México D.F., MÉxico.

2. Luke W. Sciberras, School of Mathematics and Applied Statistics, University of Wollongong, Northfields Avenue, Wollongong, New South Wales 2522, Australia.

mailto:lws31@uowmail.edu.au

3. Noel F. Smyth, School of Mathematics and Maxwell Institute for Mathematical Sciences, University of Edinburgh, Edinburgh EH9 3JZ, Scotland, U.K.

4. Annette L. Worthy, School of Mathematics and Applied Statistics, University of Wollongong, Northfields Avenue, Wollongong, New South Wales 2522, Australia. 